\title{
The impact of a toolkit on use of standardised measurement tools in stroke rehabilitation
}

Clinical Rehabilitation I-9

(C) The Author(s) 2014

Reprints and permissions:

sagepub.co.uk/journalsPermissions.nav DOI: $10.1177 / 0269215514562590$ cre.sagepub.com

(SAGE

\author{
SF Tyson', L Burton 1,2 and A McGovern²
}

\begin{abstract}
Objective: To evaluate the impact of a toolkit of psychometrically robust measurement tools, the Greater Manchester Assessment for Stroke Rehabilitation (G-MASTER) toolkit, on the use of measurement tools during stroke rehabilitation

Design: Mixed methods cohort design using non-participant observation of multi-disciplinary team meetings and semi-structured interviews with members of the team over three months before and three months after implementation of the assessment toolkit. Development and implementation of the toolkit are also described.

Setting: Ten in-patient stroke services in a large UK city.

Subjects: Members of the participating multi-disciplinary stroke teams.

Results: Before implementation standardised measures were seldom used in team meetings. After implementation, use of all measurement tools significantly increased $(36 \%$ to $81 \%$ of occasions, $P<0.000)$. Staff were generally positive about the toolkit and felt it enabled more accurate problem identification, effective progress monitoring, timely decision-making, communication and promoted inter-team relationships.

Conclusions: A toolkit of standardised measurement tools can be feasibly and acceptably implemented into stroke rehabilitation. It increases the use of measurement tools by the multi-disciplinary team and improves the processes and quality of care.
\end{abstract}

\section{Keywords}

Stroke, measurement, multi-disciplinary team

Date received: 22 August 20I4; accepted: 9 November 2014

\section{Introduction}

Standardised, objective measurement tools are recommended as a key element of assessment during stroke rehabilitation. ${ }^{1}$ Although use of such tools is prevalent, they are often unpublished and unvalidated, and uptake is haphazard. ${ }^{2}$ Health care professionals consistently report lack of consensus
IStroke Research Centre, School of Nursing, Midwifery \&
Social Work, University of Manchester UK
${ }^{2}$ Greater Manchester Strategic Clinical Network, UK

Corresponding author:

Sarah F Tyson, Stroke Research Centre, School of Nursing, Midwifery \& Social Work, Jean McFarlane Building, University of Manchester, Oxford Road, Manchester, MI 3 9PL, UK. Email: Sarah.Tyson@manchester.ac.uk 
about which measures to use, as well as lack of knowledge about how to use them, time and resources as barriers to their use..$^{2-4}$

Despite the huge literature on the psychometrics of stroke measurement tools, the make-up of assessment toolkits and their inclusion in clinical guidelines, there has been little research into the actual use of tools in clinical practice. Most investigation has considered the use of patient reported outcome measures of health related quality of life $\mathrm{e}^{5-9}$ and report that although information from these tools can improve problem detection, there is little impact on clinical management or patient outcomes. More specific to rehabilitation, several studies have considered the impact of implementing the Rehabilitation Activities Profile to provide a framework to describe patients' problems and facilitate goal setting. ${ }^{10-15}$ They also reported no change in clinical outcomes but found improvements in the processes of care, in that staff spent more time during team meetings discussing common treatment goals or management strategies rather than mere information exchange. There was also modest improvement in patients' satisfaction with their care but not on staff's satisfaction. Similar results were found when the Canadian Occupational Performance Measure was introduced in to a rheumatology rehabilitation team to facilitate goal setting; a greater emphasis on patients' needs and patient participation in rehabilitation was noted. ${ }^{16}$

Despite these promising results and the importance attached to the use of standardised measurement tools, to the authors' knowledge, the implementation of standardised measurement tools has not been examined in stroke rehabilitation. We aimed to address this issue as part of a programme of work investigating multi-disciplinary stroke rehabilitation teams. There were two foci to the programme; the structure and function of stroke rehabilitation multi-disciplinary team meetings, and the use of standardised measurement tools in these meetings. This paper reports the work to develop a toolkit of standardised, objective measurement tools for stroke rehabilitation multidisciplinary teams (called the Greater Manchester Assessment for Stroke Rehabilitation, G-MASTER) and an initial evaluation of the impact on the use of measurement tools during in-patient stroke rehabilitation. Current practice, staff views and the barriers to using standardised measurement tools have been published previously. ${ }^{2}$

Simultaneously, we explored current practice and staff views of multi-disciplinary team meetings and constructed a framework to describe the process of the meetings. We then developed and implemented an intervention to improve the quality of multi-disciplinary team meetings, called the M4 model for rehabilitation meetings (which included the G-MASTER toolkit) and evaluated the impact on the quality of multi-disciplinary team meetings and patients' outcomes. A summary of the studies and publications associated with this programme of work is shown in Supplementary Material Appendix 1. ${ }^{17-21}$

\section{Methods}

\section{Development of the G-MASTER Toolkit}

The process to develop the G-MASTER toolkit is summarised in Figure 1. Firstly, the aspects of stroke rehabilitation (referred to as domains) that needed to be measured were identified. As communication during multidisciplinary team meetings often focuses on exchange of discipline-specific information that is irrelevant or incompletely understand by other team members, ${ }^{10,11}$ it was important to identify domains that were relevant to all members of the team. To achieve this, impairments and activity limitations commonly affected by stroke were extracted from the International Classification of Functioning, Disability and Health, ${ }^{22}$ previously published core datasets ${ }^{23-25}$ and clinical guidelines. ${ }^{1}$ This list of domains was then distributed electronically to the participating stroke teams. Each team identified the domains they considered essential elements of rehabilitation that needed to be measured (rather than known or noted, such as the family situation) for all stroke rehabilitation patients, and should be known by all members of the multidisciplinary team to plan rehabilitation effectively. They also highlighted unnecessary domains and added any that had been missed. Responses were collated and a final list of domains that needed to be 


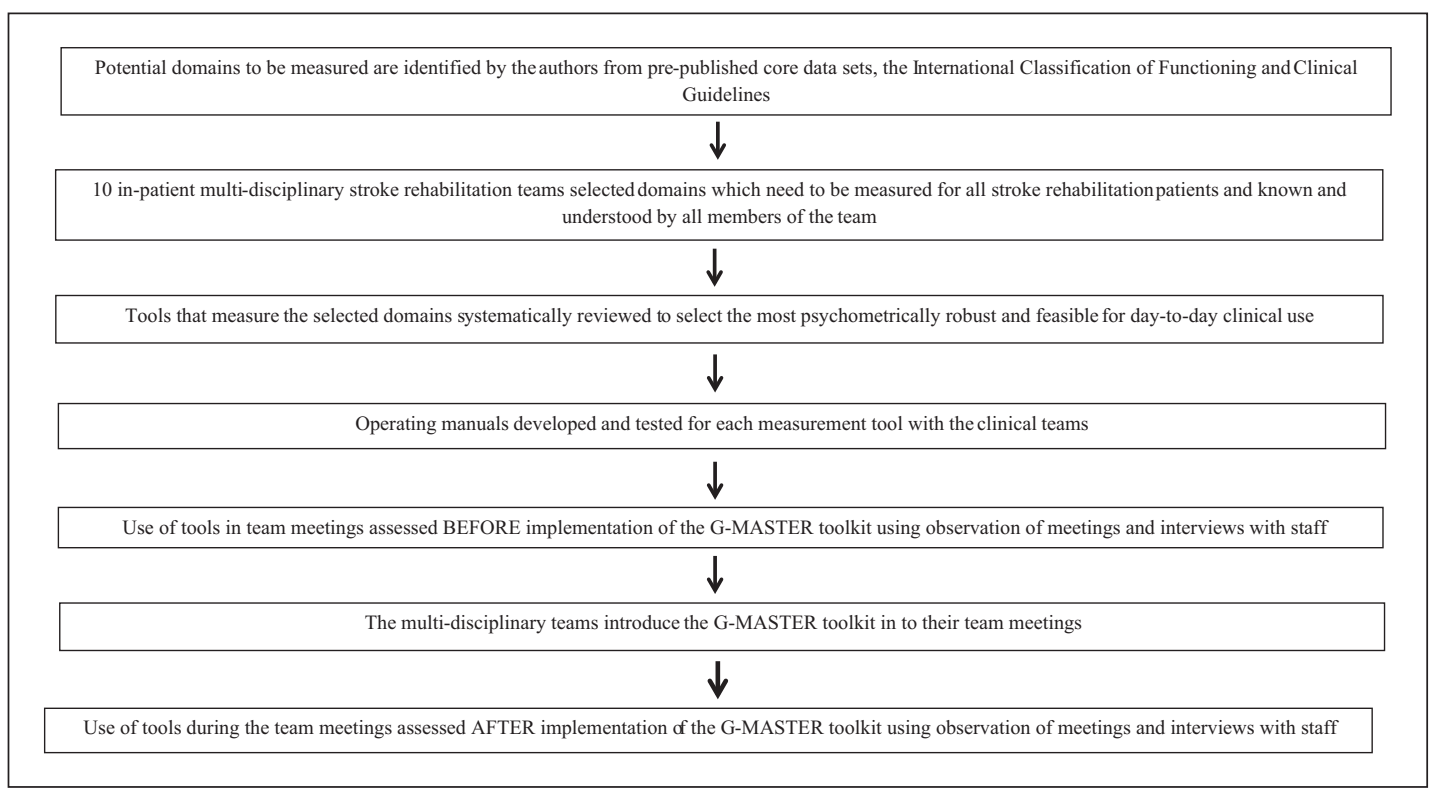

Figure I. Flow diagram illustrating the process to develop, implement and evaluate the impact of the G-MASTER toolkit.

measured was agreed at a consensus conference. The selected domains were: activities of daily living; swallowing; mood; nutritional status; cognition and communication. Continence, washing, dressing, grooming, toileting, mobility and transferring were also identified and incorporated into 'activities of daily living'.

The authors then undertook systematic reviews of the psychometric properties and clinical utility of tools that measured each domain to identify those which would produce the most robust information and could feasibly be used in day-to-day clinical practice. ${ }^{18,19}$ These were included in the toolkit. The tools selected to measure each domain were:

- Independence in the activities of daily living: Barthel Index ${ }^{26}$.

- Mood: Stroke Aphasic Depression Questionnaire (SADQ-H10) ${ }^{27}$; Patient Health Questionaire-9 (PHQ-9) ${ }^{28}$ and GAD-729 or Depression Intensity Scale Circles (DISCs) ${ }^{30}$.

- Cognition: Montreal Cognition Assessment ${ }^{31}$.

- Communication: communication section of the National Institutes of Health Stroke Scale ${ }^{32}$ or Language Screening Tool (LAST) ${ }^{33}$ (for screening) and Therapy Outcome Measures (dysphasia) (to measure outcome) ${ }^{34}$.

- Swallowing: Greater Manchester Stroke Water Swallow Screening Tool (for screening) and Therapy Outcome Measures (dysphagia) (to measure outcome) $)^{34}$.

- Nutritional Status: Malnutrition Universal Screening Tool (MUST) ${ }^{35}$.

Operating manuals were obtained for the selected tools, piloted with the participating stroke teams and amended (with further detail) as necessary. The manuals included how to administer and score the measures, timing and repetition of assessments and how they should be reported in multi-disciplinary team meetings. The operating manual containing full details of the toolkit and how it should be used is available to download. ${ }^{36}$

\section{Implementation of the G-MASTER Toolkit}

The participating stroke teams (detailed below) then started to use the toolkit in their daily practice and multi-disciplinary team meetings. As team 
meetings provides the main opportunity for teams to discuss patients and co-ordinate care, implementation of the toolkit focused on these meetings. Team members would undertake the assessments and make relevant observations during their interactions with patients. They then discussed and, where appropriate, jointly decided the patients' scores for each tool in the team meetings, and information from the scores was used to inform decision-making (such as treatment or referral decisions or discharge plans). To support this, a best practice model for the conduct of multidisciplinary team meetings (the M4 model for rehabilitation meetings) was also developed and implemented. ${ }^{20,21}$

The toolkit was implemented gradually using established service development methods such as 'Plan Do Study Act' cycles, learning sets and action planning. ${ }^{37}$ During this time, the authors regularly met with the teams to monitor progress, set and review actions, and address any difficulties.

\section{Evaluation of the G-MASTER Toolkit}

A mixed methods cohort design assessed the use of standardised measurement tools during multidisciplinary team meetings before and after implementation of the G-MASTER toolkit. Nonparticipant observation of standardised measurement tools during weekly team meetings were undertaken before and after the implementation phase. Additionally, to gain an insight in to staff's views of implementing the toolkit and the impact on clinical practice, semi-structured interviews with a purposive sample of team members (to ensure a full range of professions was represented) were undertaken during the postimplementation phase.

Selection criteria were broad. All the in-patient stroke rehabilitation teams $(n=10)$ in a large city in the UK agreed to participate which covered a range of types of hospital, services and socio-economic areas. Four were combined acute and rehabilitation units and 6 were 'stand-alone' rehabilitation units. Further details of the stroke units, models of service delivery and current practice have been published previously. ${ }^{20,21}$
Observation data were collected by one of the authors (LB) over two, three month periods; before and after implementation. At least one meeting was observed at each site at each stage, the discussion was audio-taped and field notes made. Recordings were transcribed verbatim and anonymised. To evaluate tool usage, two of the authors (LB and ST) independently analysed the meeting transcripts to identify whether the following were reported:

- scores on the identified tools, with interpretation if required

- date of assessment

- resulting actions, e.g. onward referral for further assessment

- allocation of any unreported or incomplete tools to a specific team member with time frame for completion.

Items relating to the team's overall use of the tools were also addressed. Full details are found in Table 1. For each site we ascertained whether each item was:

- always completed (score $=2$ );

- sometimes completed, but not consistently $($ score $=1)$,

- $\quad$ rarely or never completed (score $=0)$.

A score for each tool for each site was calculated by summating the scores for each item and a percentage completion rate (the score/ maximum score $\mathrm{x} 100)$ calculated to standardise the scores. A score of $100 \%$ would indicate that all the criteria for the measurement tool were met all of the time. Scores before and after implementation were compared using Mann-Whitney U tests.

Fifteen staff were interviewed, including nurses; physical, occupational and speech therapists; stroke coordinators; stroke ward managers; a psychologist and a social worker. Four were junior grades (NHS band 5); two were senior therapists (NHS Band 6); one was a highly specialist therapist (NHS band 8) and the others were in specialist posts (NHS Band 7). Length of experience in stroke care varied from a few months to over twenty years. Further details are withheld to maintain anonymity. The interviews were audiorecorded and field notes taken. The recordings 
Table I. Use of standardised measures before and after implementing the G-MASTER toolkit.

\begin{tabular}{|c|c|c|c|}
\hline & $\begin{array}{l}\text { Before implementation } \\
\text { (\% completion) }\end{array}$ & $\begin{array}{l}\text { After implementation } \\
\text { (\% completion) }\end{array}$ & $P$ value \\
\hline \multicolumn{4}{|c|}{ Use of Barthel Index to assess independence in the activities of daily living } \\
\hline Patients' score stated & $3(18 \%)$ & $13(81 \%)$ & 0.007 \\
\hline All relevant members contribute to scoring & $4(24 \%)$ & $10(63 \%)$ & 0.161 \\
\hline Standardised documentation used to record scores & $4(24 \%)$ & $12(75 \%)$ & 0.105 \\
\hline Scores compared to the previous week & $\mathrm{I}(6 \%)$ & $9(54 \%)$ & 0.021 \\
\hline Total $($ maximum score $=64)$ & $12(19 \%)$ & $44(69 \%)$ & 0.00 \\
\hline \multicolumn{4}{|c|}{ Use of Therapy Outcome Measures to assess language and swallowing } \\
\hline Patients with language difficulties scored & $0(0 \%)$ & $12(75 \%)$ & 0.000 \\
\hline Patients with swallowing difficulties scored & $0(0 \%)$ & $13(81 \%)$ & 0.000 \\
\hline Scores compared to previous weeks & $0(0 \%)$ & $4(24 \%)$ & 0.234 \\
\hline Total $($ maximum score $=48)$ & $0(0 \%)$ & $29(60 \%)$ & 0.000 \\
\hline \multicolumn{4}{|l|}{ Use of standardised screening tools to assess mood } \\
\hline Patients' score stated & $2(13 \%)$ & $9(54 \%)$ & 0.021 \\
\hline Date of completion stated & $\mathrm{I}(6 \%)$ & $\mathrm{I}(6 \%)$ & I \\
\hline Interpretation of screen stated & $2(13 \%)$ & $10(63 \%)$ & 0.015 \\
\hline Action as a result of screen stated & $\mathrm{I}(6 \%)$ & $8(48 \%)$ & 0.009 \\
\hline $\begin{array}{l}\text { If incomplete, completion is allocated to a specific } \\
\text { member with a deadline }\end{array}$ & $0(0 \%)$ & $6(36 \%)$ & 0.038 \\
\hline Total (maximum score $=80$ ) & $6(8 \%)$ & $34(43 \%)$ & 0.000 \\
\hline \multicolumn{4}{|c|}{ Use of Montreal Cognitive Assessment to assess cognition } \\
\hline Patients' scores stated & $\mathrm{I}(6 \%)$ & $6(36 \%)$ & 0.083 \\
\hline Date of completion stated & $0(0 \%)$ & $0(0 \%)$ & 1 \\
\hline Interpretation stated & $\mathrm{I}(6 \%)$ & $9(54 \%)$ & 0.065 \\
\hline Action as a result of screen stated & $0(0 \%)$ & $3(18 \%)$ & 0.189 \\
\hline $\begin{array}{l}\text { If incomplete, completion is allocated to a specific } \\
\text { member with a deadline }\end{array}$ & $0(0 \%)$ & $6(36 \%)$ & 0.234 \\
\hline Total $($ maximum score $=80$ ) & $2(3 \%)$ & $24(30 \%)$ & 0.000 \\
\hline \multicolumn{4}{|c|}{ Use of Malnutrition Universal Screening Tool to assess nutrition } \\
\hline Patients' scores stated & I (6\%) & $10(63 \%)$ & 0.005 \\
\hline Date of completion stated & $0(0 \%)$ & $0(0 \%)$ & I \\
\hline Interpretation stated & $0(0 \%)$ & $7(42 \%)$ & 0.038 \\
\hline Action as a result of screen stated & $0(0 \%)$ & $8(48 \%)$ & 0.038 \\
\hline $\begin{array}{l}\text { If incomplete, completion is allocated to a specific } \\
\text { member with a deadline }\end{array}$ & $0(0 \%)$ & $2(12 \%)$ & 0.721 \\
\hline Total (maximum score $=80$ ) & $\mathrm{I}(\mathrm{l} \%)$ & $27(33 \%)$ & 0.000 \\
\hline \multicolumn{4}{|l|}{ Team's overall use of standardised measures in meetings } \\
\hline $\begin{array}{l}\text { Scores and dates of completed assessments } \\
\text { reported }\end{array}$ & 2 & 13 & 0.001 \\
\hline Members can interpret scores & 4 & 9 & 0.065 \\
\hline Scores are related to the patient's goals & 0 & 4 & 0.442 \\
\hline $\begin{array}{l}\text { Team members bring information to score } \\
\text { measures during the meeting }\end{array}$ & 2 & 13 & 0.021 \\
\hline Total (maximum score $=64$ ) & $8(13 \%)$ & $39(61 \%)$ & 0.000 \\
\hline All items combined (maximum score $=416$ ) & $161(39 \%)$ & $213(51 \%)$ & 0.000 \\
\hline
\end{tabular}

$\%$ completion: how often each item was completed in the observed multidisciplinary team meetings, $100 \%$ : item was addressed for all patients in all observed meetings, $0 \%$ : the item was never addressed. 
were transcribed verbatim and anonymised for person and site. Data collection methods and analysis have been described previously, ${ }^{36}$ but in summary we used inductive thematic content analysis to identify staff's views about the toolkit; effectiveness, benefits and barriers to success and how it could be improved.

Although we sought ethical approval from the National Research Ethics Service, their view was that the project did not require approval. All participating staff gave consent for data collection.

\section{Results}

Before implementation of the G-MASTER toolkit, standardised measures were seldom used in team meetings (overall score $=38 \%$, Table 1 ). Measures of communication, swallowing, cognition and nutrition were never or very rarely discussed. The Barthel Index (a measure of independence of the activities of daily living) was reported most often but only two sites did so consistently (overall score $=19 \%$ ). After implementation, use of all measurement tools significantly increased $(36 \%-81 \%$, $P<0.000$, Table 1).

The interviews showed that staff were generally positive about the toolkit. The most frequently noted change was that it enabled patients' progress to be objectively monitored. As a physiotherapist explained: "[We] would not want to just make an assumption about, or treat on gut feeling, so to have a bit of an objective marker helps the team focus".

Objective information also facilitated communication with patients' families about progress and future plans: "I was reluctant to do the Barthel every week, because I didn't know what it would add, but actually in its own way, for those patients are not achieving at all, it can be a really useful tool to demonstrate to patients and relatives". [Occupational Therapist].

Staff felt that objective measures enabled them to monitor treatment plans and rehabilitation goals which informed planning and decision-making by providing structure and prompted them "to think" [Occupational Therapist] comprehensively. An additional benefit was that it enabled "more proactive"
[Physiotherapist] identification of higher level difficulties that were missed before, which prompted more timely treatment or referrals.

There was also an impact on the team climate. Staff reported that discussion around the scores enhanced awareness and understanding of the patients' difficulties and each disciplines' work to manage them. This built camaraderie: "Understanding each other's scores, which I think we're starting to do, that's what's helpful". [Speech therapist]

These effects were not instantaneous however; it took time and training for the teams to become familiar with the measures and their interpretation. However once embedded, they "became second nature" providing a common language that facilitated discussion and decision-making: "Everyone understands [the tools] and that gives us the opportunity to understand the patient's deficit and quickly work out management strategies, which helps discharge planning. I think our throughput is quick now, ..... we are getting to grips with patients' difficulties, managing them and moving forward to discharge more efficiently than we were". [Stroke co-ordinator]

\section{Discussion}

These results indicate that a toolkit of psychometrically robust standardised measurement tools can be implemented feasibly and acceptably into inpatient stroke rehabilitation and improves the usage of measurement tools by multi-disciplinary teams. It can also improve the effectiveness of team meetings and quality of care, in that staff reported the toolkit enabled more accurate problem identification, effective progress monitoring, timely decision-making, communication and promoted inter-team relationships. Furthermore the increased use of standardised measurement tools would enable recommendations in national clinical guidelines to be met; a proxy marker of service quality. ${ }^{1}$ These results compliment quantitative reports that use of standardised measures can improve the process of care. However, our use of both quantitative and qualitative methods gives 
greater detail and insight in to possible mechanisms for the improvements seen.

Although significant improvements in measurement tool usage were noted, adherence to the toolkit was still sub-optimal in some areas, particularly the assessment of mood, cognition, nutrition and for monitoring scores from week to week. This may be a reflection of the lack of psychologists and dieticians in most participating multi-disciplinary teams; however the selected measurement tools could be administered by any member of the team. Alternatively, professional tribalism may have made some staff reluctant to engage in work they considered the remit of other professions. This suggests that although staff noted the tools promoted inter-team understanding and a focus on patients' problem rather than professional activity, there is still some way to go before some teams function in an inter-disciplinary 'patient-centred' manner. Furthermore, we used best-evidence implementation and service improvement strategies to promote change in practice, ${ }^{38-40}$ but like other authors we found it had limited success in some cases. $^{40-42}$ Further work is needed to establish clinically and cost-effective ways to improve service delivery, particularly for complex interventions delivered by multi-disciplinary teams as most work to date has focused on changing prescribing practice by doctors. ${ }^{40}$

Our encouraging findings came from an uncontrolled cohort design so the observed changes cannot be solely attributed to the G-MASTER toolkit (although we are unaware of other work which would induce such changes). Furthermore, assessments could not blinded; staff knew they were being assessed and the assessors were involved in the development and implementation of the intervention which may have produced reporting bias and/or a Hawthorne effect. Nevertheless, our results indicate that a feasible, acceptable toolkit of measurement tools for stroke rehabilitation can be implemented and may impact on clinical practice. Clinical trials are warranted to investigate the impact of the G-MASTER toolkit on outcomes using more robust trial designs.

\section{Clinical messages}

- Stroke rehabilitation professionals consider that the activities of daily living, mood, cognition, communication, nutrition and swallowing should be measured for all patients and be understood by all members of the team.

- The G-MASTER toolkit enabled more efficient identification of patients' problems; progress, monitoring; timely decision-making, whilst promoting communication and inter-team relationships, and significantly increased the use of standardised measurement tools.

\section{Conflict of interest}

The authors declare that there is no conflict of interest.

\section{Funding}

This project has been funded through a Knowledge Transfer Partnership (grant number 0007812), funded by the Technology Strategy Board and the Greater Manchester \& Cheshire Cardiac and Stroke Network.

\section{References}

1. Intercollegiate Stroke Working Party. National Clinical Guideline for stroke, 4th Ed. London: Royal College of Physicians, 2012.

2. Burton L, Tyson SF and McGovern A. Staff perceptions of using outcome measures in stroke rehabilitation. Disab \& Rehab 2013; 35: 828-834.

3. van Peppen R, Maissan F, van Genderen F, et al. Outcome measures in physiotherapy for patients with stroke: a survey into self-reported use, and barriers to and facilitators for use. Physioth Res Int 2008; 13: 255-270.

4. Fung $\mathrm{CH}$ and Hays RD. Prospects and challenges in using patient-reported outcomes in clinical practice. Qual Life Res 2008; 17: 1297-1302.

5. Greenhalgh J. The applications of Patient Reported Outcomes in clinical practice: what are they, do they work, and why? Qual Life Res 2009; 18: 115.

6. Valderas JM, Kotzeva A, Espallargues M, et al. The impact of measuring patient-reported outcomes in clinical practice: A systematic review of the literature. Qual Life Res 2008; 17: 179-193.

7. Marshall S, Haywood KL and Fitzpatrick R. Impact of patient-reported outcome measures on routine practice: A structured review. J Eval Clin Pract 2006; 12: 559-568. 
8. Gilbody SM, Whitty PM, Grimshaw JM, et al. Improving the detection and management of depression in primary care. Qual Saf Health Care 2003; 12: 149-155.

9. Greenhalgh $J$ and Meadows K. The effectiveness of the use of patient-based measures of health in routine practice in improving the process and outcomes of patient care: A literature review. J Eval Clin Pract 1999; 5: 401-416.

10. Jelles F, van Bennekom CAM, Lankhorst GJ, et al. Introducing an innovative method in team conferences. Disabil Rehabil 1996; 18: 374-379.

11. Jelles F, van Bennekom CAM and Lankhorst GJ. The interdisciplinary team conference in rehabilitation medicine. Am J Phys Med Rehabil 1995; 74: 464-465.

12. Jelles F, van Bennekom CAM and Lankhorst GJ. Instruments to structure interdisciplinary team conferences in rehabilitation medicine: the added value of the Rehabilitation Activities Profile. J Rehabil Sci 1995; 8: 51-57.

13. Verhoef J, Toussaint PJ, Zwetsloot-Schonk JHM, et al. Effectiveness of the introduction of an International Classification of Functioning, Disability and Healthbased rehabilitation tool in multidisciplinary team care in patients with rheumatoid arthritis. Arthrit \& Rheum 2007; 57: 240-248.

14. Verhoef J, Toussaint PJ, Putter H, et al. The impact of the implementation of a rehabilitation tool on the contents of the communication during multidisciplinary team conferences in rheumatology. Int J Med Info 2007; 76: 856-863.

15. Verhoef J, Toussaint PJ, Putter H, et al. The impact of introducing an ICF-bas10-13ed rehabilitation tool on staff satisfaction with multidisciplinary team care in rheumatology: an exploratory study. Clin Rehabil 2008; 22: 23-37.

16. Wressle E, Lindstrand J, Neher M, et al. The Canadian Occupational Performance Measure as an outcome measure and team tool in a day treatment programme. Disab \& Rehabil 2003; 25: 497-506.

17. Tyson S, Burton L and McGovern A. The impact of an assessment toolkit on use of objective measurement tools in stroke rehabilitation. (Submitted) Clin Rehab.

18. Burton L and Tyson SF. Screening for mood disorders after stroke: A systematic review of psychometric properties and clinical utility. Psychol Med. Epub ahead of print 28 February 2014. DOI:10.1017/S0033291714000336.

19. Burton L and Tyson SF. Screening for cognitive impairment after stroke: A systematic review of psychometric properties and clinical utility. J Rehab Med 2014. DOI:10.2340/16501977-1930.

20. Tyson SF, Burton L and McGovern A. Multi-disciplinary team meetings in stroke rehabilitation: An observation study. Clin Rehab. Epub ahead of print 4 June 2014. DOI: $10.1177 / 026921551453594$.

21. Tyson SF, Burton LJ and McGovern A. The effect of structured model for stroke multi-disciplinary team meetings on service delivery and patient outcomes. (Submitted) Clin Rehab.
22. World Health Organisation. International Classification of Functioning, Disability and Health. Geneva: World Health Organisation, 2001.

23. Tyson S, Watson A, Moss S, et al. Development of a framework for the evidence-based choice of outcome measures in neurological physiotherapy. Disabil Rehabil. 2008; 30: 142-149.

24. Glässel A, Coenen M, Kollerits B, et al. Validation of the extended ICF core set for stroke from the patient perspective using focus groups. Disabil Rehabil 2012; 34: 157-166.

25. Southwest Ontario Stroke Strategy Group. Stroke Rehabilitation Outcome Measures Forum. 2009. Available at: http:/www.ontariostrokenetwork.ca/ wp-content/uploads/2013/09/SWO_Stroke_Rehab_ Outcome_Measures_Forum_2009.pdf.

26. Collin C, Wade DT, Davies S, et al. The Barthel ADL Index: A reliability study. Disab \& Rehabil 1998; 10: 61-63.

27. Lincoln NB, Sutcliffe LM and Unsworth G. Validation of the Stroke Aphasic Depression Questionnaire (SADQ) for use with patients in hospital. Clin Neuropsych Assess 2000; 1: 88-96.

28. Spitzer RL, Kroenke K, Williams JBW, et al. A brief measure for assessing generalised anxiety disorder (GAD7). Arch Intern Med 2006; 166: 1092-1097.

29. Spitzer R, Kroenke K and Williams J. Validation and utility of a self-report version of PRIME-MD: The PHQ primary care study. JAMA 1999; 282; 1737-1744.

30. Turner-Stokes L, Kalmus M, Hirani D, et al (2005). The Depression Intensity Scale Circles (DISCs): a first evaluation of a simple assessment tool for depression in context of brain injury. J Neurol Neurosurg Psych 2005; 76: 1273-1278.

31. Nasreddine ZS, Phillips NA, Bédirian V, et al. The Montreal Cognitive Assessment (MoCAC): A Brief Screening Tool for Mild Cognitive Impairment. $J$ Am Geriatr Soc 2005; 53: 695-699.

32. National Institute of Health, National Institute of Neurological Disorders and Stroke. Stroke Scale. Available at: http://www.ninds.nih.gov/doctors/NIH_ Stroke_Scale.pdf.

33. Flamand-Roze C, Falissard B, Roze E, et al. Validation of a new language screening tool for patients with acute stroke: The Language Screening Test (LAST). Stroke 2011; 42: 1224-1229.

34. Enderby P, John A and Petheram B. (2006). Therapy Outcome Measures for Rehabilitation Professionals (2nd $E d)$. Wiley: UK.

35. Elia M (2003) Screening for malnutrition: A multidisciplinary responsibility. Development and use of the Malnutrition Universal Screening Tool ('MUST') for adults. Redditch: BAPEN.

36. Tyson SF, Burton LJ and McGovern A. The Greater Manchester Assessment for Stroke Rehabilitation. 
Available at: https://www.escholar.manchester.ac.uk/ukac-man-scw:230101 (accessed 31 July 2014).

37. Nakayama DK, Bushey TN, Hubbard I, et al. Using a Plan-Do-Study-Act Cycle to Introduce a New OR Service Line. ORN Journal 2010; 92: 335-343.

38. Bosch M, van der Weyden $\mathrm{T}$, Wensing $\mathrm{MG}$, et al. Tailoring quality improvement interventions to identified barriers: a multiple case analysis. J Eval Clin Pract. 2007; 13: 161-168.

39. Grol R and Grimshaw JM. From best evidence to best practice: effective implementation of change in patients' care. Lancet 2003; 362: 1225-1230.
40. Baker R, Camosso-Stefinovic J, Gillies C, et al. Tailored interventions to overcome identified barriers to change: effects on professional practice and health care outcomes. Cochrane Database of Systematic Reviews 2010: 3: CD005470.

41. Grimshaw JM, Thomas RE, MacLennan G, et al. Effectiveness and efficiency of guideline dissemination and implementation strategies. Health Technology Assessment, 2004; 8:6: 1-72.

42. Chaillet N, Dube E, Dugas M, et al. Evidence-based strategies for implementing guidelines in obstetrics. Obs \& Gynec 2006; 108: 1234-1245. 